\title{
FULL POLARIMETRIC UAVSAR IMAGE CHANGE DETECTION BASED ON CHANGE INDICES
}

\author{
Leila Yousefizadeh ${ }^{\mathrm{a}}$, Reza Shahhoseini ${ }^{\text {a*}, \text { Saeid Homayouni }}{ }^{\mathrm{b}}$ \\ ${ }^{a}$ School of Surveying and Geospatial Engineering, College of Engineering, University of Tehran, Tehran, Iran, \\ (leila.yousefizade, rshahosseini@ut.ac.ir) \\ b Centre Eau Terre Environment, INRS-Quebec, Canada, saeid.homayouni@ete.inrs.ca
}

KEY WORDS: Unsupervised Change Detection, UAVSAR, Change Index, Kernel K-means

\begin{abstract}
:
Change detection is one of the most important applications of Polarimetric Synthetic Aperture Radar (PolSAR) data in monitoring urban development and supporting urban planning due to the sensibility of SAR signal to geometrical and physical properties of terrestrial features. In this paper, we proposed an unsupervised change detection method using change indices extracted from PolSAR images. Kernel k-means clustering was then performed to extract changed areas. The kernel k-means clustering is an unsupervised algorithm that maps the input features to higher Hilbert dimension space by using a kernel function. To better representation of changed areas, different change indices were generated. The method was applied to UAVSAR L-band SAR images acquired over an urban area in San Andreas, United States. We evaluated the change detection performance based on kappa and overall accuracies of the proposed approach and compared with other well-known classic methods.
\end{abstract}

\section{INTRODUCTION}

Image change detection is a process that analyses images of the same scene taken at different times in order to identify changes that may have occurred between the considered acquisition dates (Radke et al. 2005). Developing SAR imaging system and its capability to image Earth in both day and night, and for almost all weather conditions, make it possible to use this system in detecting changed areas, urban expansion monitoring, agricultural and forest studies (Lee and Pottier 2009). Polarimetric SAR sensor measures the amplitude and phase of backscattered signals in four combinations of the linear receive and transmits polarizations: $\mathrm{HH}, \mathrm{HV}, \mathrm{VH}$, and $\mathrm{VV}$ which increases discrimination ability to extract different change type as compared with single- polarization (Conradsen et al. 2003) Change detection methods can be divided into two categories: supervised and unsupervised. Supervised CD methods need prior knowledge of the area wherein changes have happened for training algorithms, such as support vector machine (SVM) and artificial neural network (ANN), while in unsupervised methods there is no need to have a priori knowledge; this is useful when training data is unavailable. Unsupervised CD methods are achieved with three steps: 1) preprocessing, which includes coregistration and noise reduction; 2) producing difference image which can be made by subtraction operator or ratio operator, and 3) analysing difference image to discriminate between changed and unchanged area. In the third step, changed areas are detected by several methods such as: thresholding (Moser and Serpico 2006) (Bazi, Bruzzone, and Melgani 2005), clustering (Celik 2009) (Gong et al. 2013), etc. Several thresholding methods have been proposed such as (Otsu 1979), (Kittler and Illingworth 1986), expectation-maximization (EM) (Sezgin and Sankur 2004). However, finding appropriate global threshold depends on the statistical characteristics of the difference images which are often unknown (Melgani and Bazi 2006). A possible approach to solve this problem is to use clustering methods used to classify feature vectors into "changed" and "unchanged" class. (Celik 2009) used k-means clustering on the principal component analysis (PCA) of the difference image to extract change map. (Volpi et al. 2010) introduced a kernel k-means approach solving the problem of data's nonlinearity by representing the difference image in the feature space using kernel functions. Also, In order to have a soft clustering, fuzzy c-means (FCM) were used in ( $\mathrm{Li}$ et al. 2015) to classify the log-ratio difference image.

In the SAR data processing, it is common to introduce a test statistic and apply a decision threshold to extract the change map. (Conradsen et al. 2003) presented the Wishart likelihood ratio test (LRT) as a new test statistic for change detection in multilook PolSAR images. (Akbari et al. 2013) utilized the complex-kind Hotteling-Lawley trace (HLT) as a test statistic for change detection in multilook PolSAR images.

In this paper, we proposed a method based on a fusion of different change indices like log-ratio on intensity, log-ratio on polarimetric decomposition and using a test statistic based on Wishart distribution as a change index to use them as an input of kernel k-means clustering.

This paper is organized as follows. Section 2 describes the proposed unsupervised change detection approach. Section 3 describes the study area and data sets. Section 4 provides experimental results on real multitemporal SAR images and Section 5 is the conclusion.

\section{PROPOSED METHOD}

In change detection, two co-registered SAR images $\mathrm{X}_{1}$ and $\mathrm{X}_{2}$ with a size of $\mathrm{M} \times \mathrm{N}$ over the same area at different times are considered as input data. The procedure of the proposed method is illustrated in Fig. 1. It mainly consists of the following three steps.

\footnotetext{
* Corresponding author
} 
Step 1) preprocessing: In speckle reduction step, Lee refined filter with a $5 \times 5$ window size can be used to suppress speckle noise effect.

Step 2) feature level fusion: The aim of fusion at the feature level of multi-temporal images is to generate new features that highlight multi-temporal information. These features are often referred to as change indices $(\mathrm{CI})$ since they are employed to highlight changes occurred in bi-temporal image pairs (Bovolo and Bruzzone 2015).

Step 3) clustering: This step aims at Portioning the CI into two classes of changed and unchanged by using unsupervised classification. The CI, which is obtained from Step 2, is considered as input in this step.

\subsection{Feature Level Fusion}

Several mathematical operators can be applied to extract a change index. In SAR images processing, the log-ratio operator is preferred because it can reduce the multiplicative components of errors and enhance the low-intensity pixels.

$$
\mathrm{CI}=\left|\log \left(\frac{X 2}{X 1}\right)\right|=|\log (X 2)-\log (X 1)|
$$

In Polarimetric SAR, change indices can be generated after extracting specific features such as the backscattering coefficient and polarimetric decomposition. Moreover, due to the more precious information of polarimetric data, additional change indices based on the covariance matrix such as the test of equality for two complex Wishart matrixes can be considered.

Test Statistic for the Equality of Two Covariance Matrices: We consider that the two covariance matrices $\left(C_{1}, C_{2}\right)$ from the bitemporal PolSAR images are independent and follow a Wishart distribution as follows:

$$
\begin{aligned}
& C_{1} \in W\left(p, m, \sum C_{1}\right) \\
& C_{2} \in W\left(p, n, \sum C_{2}\right)
\end{aligned}
$$

Where $p$ represents the dimensions of $C 1$ and $C 2$, and $m$ and $n$ represent the number of looks of $C 1$ and $C 2$, respectively. $\Sigma C 1$ and $\Sigma C 2$ represent the dispersion matrix of $C 1$ and $C 2$, and the (maximum likelihood estimation) MLE of $\Sigma C 1$ and $\Sigma C 2$ can be shown as follows:

$$
\sum_{C_{1}}^{M L E}=\frac{1}{m} C_{1} \text { and } \sum_{C_{2}}^{M L E}=\frac{1}{n} C_{2}
$$

Assuming that the null hypothesis $H 0$ : $\sum C 1=\sum C 2$ means that the two matrices are equal and there is a strong possibility of nonchange, the alternative hypothesis $H 1: \sum C 1 \neq \sum C 2$ means that the two matrices are different and there is a strong possibility of change (Conradsen et al. 2003).

We suppose that the test statistics based on MLE have joint densities $f\left(\sum C 1, \quad \sum C 2, \quad \theta\right)$, where $\theta$ is the set of parameters of the probability function that has generated the data. Then $H 0$ states that $\theta \in H 0$, and the likelihood ratio of the test statistic is shown as follows:

$$
Q=\frac{\max _{\theta \in \mathrm{H}_{0}} L(\theta)}{\max _{\theta \in \Omega} L(\theta)}
$$

where $L(\theta)=f\left(\sum_{C_{1}}, \sum_{C_{1}}, \theta\right)=f\left(\sum_{C_{1}}, \theta\right) f\left(\sum_{C_{2}}, \theta\right)$

Where $\Omega=H 0 \cup H 1, L(\cdot)$ is the likelihood function, and $f(\cdot)$ is the frequency function.

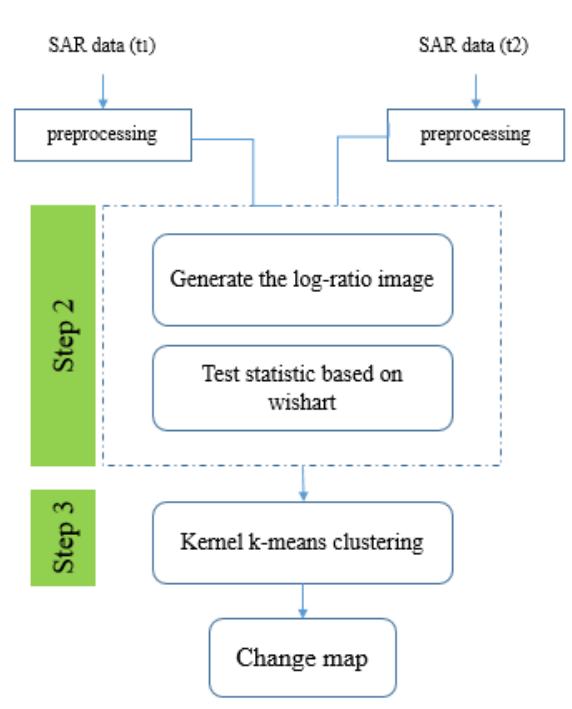

Fig. 1. Flowchart of the proposed method

The MLE of Equation (5) can be simplified as follows:

$$
Q=\frac{L(\bar{\Sigma})}{L_{C_{1}}\left(\bar{\Sigma}_{C_{1}}\right) L_{C_{2}}\left(\bar{\Sigma}_{C_{2}}\right)}=\frac{(n+m)^{p(n+m)}}{n^{p n} m^{p m}} \frac{\left|C_{1}\right| n\left|C_{2}\right|^{m}}{\left|C_{1}+C_{2}\right|^{n+m}}
$$

Assuming $m=n$, the CI of the bi-temporal PolSAR images can be denoted by $d$ :

$$
\begin{aligned}
d=-2 \rho \ln Q=- & 2 \rho n\left(2 p \ln 2+\ln \left|\mathrm{C}_{1}\right|+\ln \left|C_{2}\right|\right. \\
& \left.-\ln \left|C_{1}+C_{2}\right|\right)
\end{aligned}
$$

Where $\rho$ can be represented as follows:

$$
\rho=1-\frac{2 p^{2}-1}{6 p}\left(\frac{1}{n}+\frac{1}{m}-\frac{1}{n+m}\right)
$$

\subsection{Clustering}

Clustering is the assignment of a set of observations into subsets (called clusters) so that observations in the same cluster are similar in some sense. One of the mainly used clustering algorithms is k-means clustering which partitions the given data into $k$ clusters by randomly choosing $\mathrm{k}$ centroid, and minimizes the distance between data and centroid:

$$
d^{2}\left(x_{i}, m_{k}\right)=\left\|x_{i}-m_{k}\right\|^{2}
$$

Where xi $\in X$ input data and $m_{k}=\frac{1}{|\pi k|} \sum_{j \epsilon \pi_{k}} x j, \pi_{k}$ is the elements assigned to cluster $\mathrm{k}$, and $\left|\pi_{k}\right|$ is their number.

\subsubsection{Kernel K-means Clustering}

The leading idea of kernel methods is that nonlinear decision rules can be achieved by running a linear algorithm in a higher dimensional feature space, the reproducing kernel Hilbert space (RKHS), where the solution is more likely to be linear (Volpi et al. 2010). The mapping function is commonly represented as $\varphi($.$) . In practice, it is usually hard to determine the mapping$ function and often impossible to compute directly the sample coordinates in the Hilbert space. The mapping to that space is implicitly defined by kernel functions replacing dot products in 
the original formulation, only needing input samples in their original space. Using mapped samples, the k-means becomes:

$$
d^{2}\left(\varphi\left(x_{i}\right), m_{k}\right)=\left\|\varphi\left(x_{i}\right)-m_{k}\right\|^{2}
$$

Where. $\quad m_{k}=\frac{1}{|\pi k|} \sum_{j \epsilon \pi_{k}} \varphi(x j)$

This is equivalent to:

$$
\begin{aligned}
d^{2}\left(\varphi\left(x_{i}\right), m_{k}\right)= & \left\langle\varphi\left(x_{i}\right), \varphi\left(x_{i}\right)\right\rangle+\left\langle m_{k}, m_{k}\right\rangle \\
& -2\left\langle\varphi\left(x_{i}\right), m_{k}\right\rangle
\end{aligned}
$$

By plugging (11) into (12), and replacing the dot product $\langle\varphi(),. \varphi()$.$\rangle by a proper kernel function k(.,$.$) , the kernel \mathrm{k}-$ means formulation is obtained as (Shawe-Taylor and Cristianini 2004):

$$
\begin{aligned}
d^{2}\left(\varphi\left(x_{i}\right), m_{k}\right)= & \left\langle\varphi\left(x_{i}\right), \varphi\left(x_{i}\right)\right\rangle \\
& +\frac{1}{|\pi k|^{2}} \sum_{j, m \epsilon \pi_{k}}\left\langle\varphi\left(x_{i}\right), \varphi(m)\right\rangle \\
& -\frac{1}{|\pi k|} \sum_{j, \epsilon \pi_{k}}\left\langle\varphi\left(x_{i}\right), \varphi\left(x_{j}\right)\right\rangle \\
& =k\left(x_{i}, x_{i}\right) \\
& +\frac{1}{|\pi k|^{2}} \sum_{j, m \epsilon \pi_{k}} k\left(x_{i}, x_{m}\right) \\
& -\frac{2}{|\pi k|} \sum_{j, \epsilon \pi_{k}} k\left(x_{i}, x_{j}\right) .
\end{aligned}
$$

\section{STUDY AREA AND DATASET}

In this letter, we used two images acquired by full polarimetry Uninhabited Aerial Vehicle Synthetic Aperture Radar (UAVSAR) in L-band over an urban area in San Andreas city on October 23, 2014, and January 21, 2019. In order to give a detailed assessment, the dataset was separated into two regions. The first region mainly involves buildings $(150 \times 200$ pixels $)$ and the second one involves vegetation $(260 \times 160$ pixels). Fig. 2 and Fig. 3 show the two regions and the manually defined ground truth.

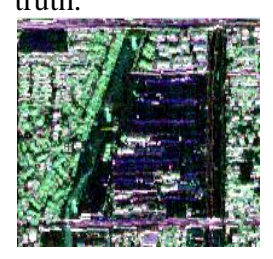

a

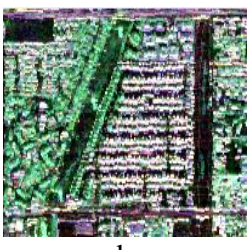

b

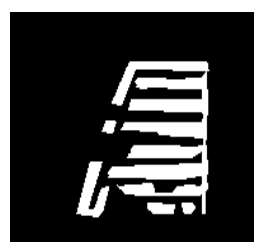

c

Fig. 2. Pauli images of dataset, (a) Region 1 in 2014, (b) Region 1 in 2019, (c) ground truth Region 1.

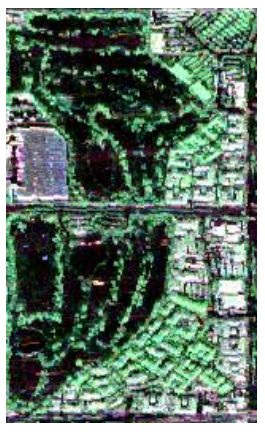

a

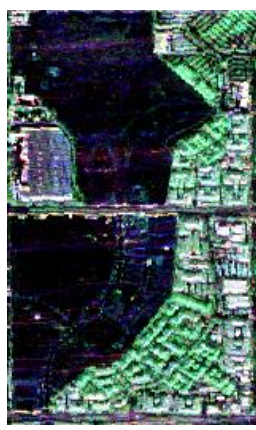

b

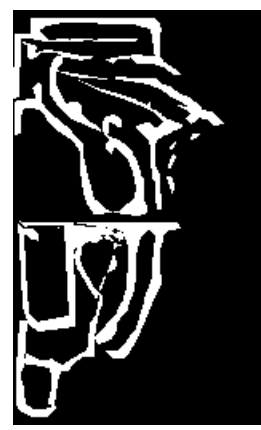

Fig. 3. Pauli images of dataset, (a) Region2 in 2014, (b) Region 2 in 2019, (c) ground truth Region 2.

\section{RESULTS}

According to the illustrated flowchart in Fig. 1, after performing pre-processing into two steps, for each image, different features were extracted and grouped into two categories of, intensity and polarimetric decomposition. Then, the log-ratio operator was applied on features to produce change indices. In the next step, we used produced change indices and the change index based on a test statistic for the equality of two covariance matrices as an input of the kernel k-means clustering. The kernel k-means clustering requires careful tuning of the parameters of the kernel function. For an RBF kernel, we had to choose different scale parameters $\sigma=\left\{10^{-3}, 10^{-2}, \ldots, 6\right\}$ in order to obtain reasonable results. To evaluate the efficiency of our proposed method, comparative experiments were designed including implementation of our proposed method, using k-means clustering, and apply a test statistic based on Wishart distribution. In order to have a quantitative evaluation, kappa coefficient and overall accuracy $(\mathrm{OA})$ were used.

\subsection{Results on the Build-up area}

In region 1, there is a strong response in all types of change indices. Because this area consists of dense buildings, which make a high value in different polarizations both in crosspolarization and co-polarization response, as shown in Fig. 4. In the build-up area, double-bounce scattering is the main scattering mechanism; however, the density of an urban area also contributes to volume scattering. Therefore, in this area, scattering mechanisms is a combination of a surface, volume, and double-bounce scattering. Therefore, the change indices were obtained by model-based polarimetric decomposition can extract constructed buildings. Fig. 5

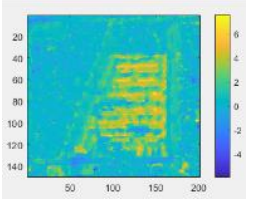

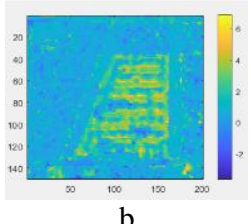

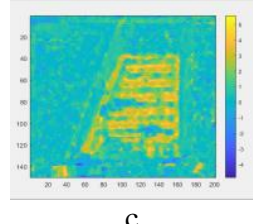

Fig. 4. Change indices in region 1 based on, (a) log-ratio $\mathrm{HH}$, (b) log-ratio VV, (c) log-ratio VH

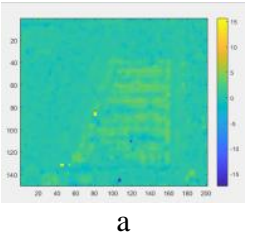

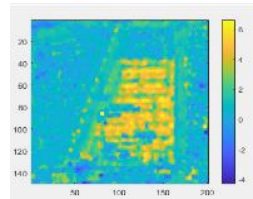

$\mathrm{b}$

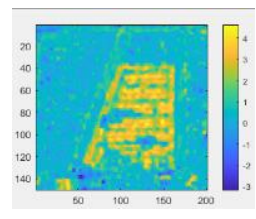

$\mathrm{c}$
Fig. 5. Change indices in region 1 based on, (a) log-ratio Yamaguchi Odd, (b) log-ratio Yamaguchi Dbl, (c) log-ratio Yamaguchi Vol

Fig. 6 shows the change maps obtained by different methods. According to Table 1 Performance evaluation of the change detection, the proposed method has the highest OA. Using multiple features related to the structure of the build-up area, and applying the kernel k-means clustering with a nonlinear boundaries leads to better performance. On the other hand, as shown in Fig. 6 (c) the result of the k-means clustering contains many wrong detections in unchanged areas in the background. We can understand due to the nonlinear nature of the change 
indices; linear partitioning has a poor performance. The result of the test statistic shows that this method has the capability to extract changed areas accurately.

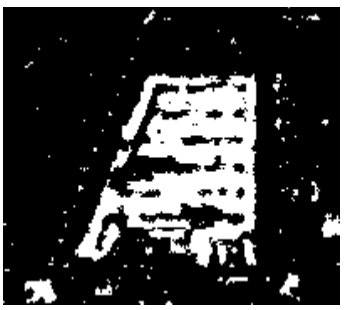

a

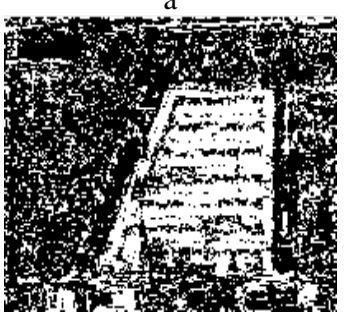

$\mathrm{c}$

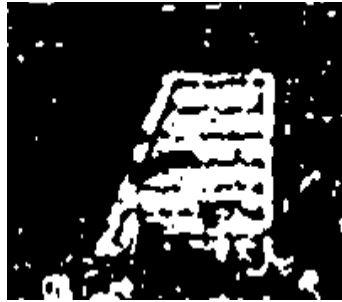

b

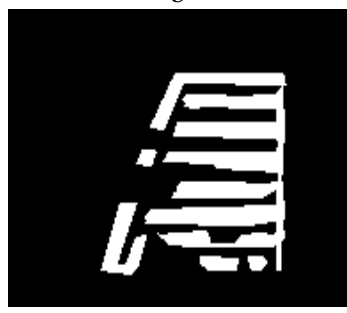

d
Fig. 6. Change detection results in Region 1, (a) proposed method, (b) test statistic based on Wishart distribution, (c) kmeans clustering, (d) ground truth

Table 1 Performance evaluation of the change detection

\begin{tabular}{ccc}
\hline Method & OA (\%) & Kappa \\
\hline Kernel k-means & 89.82 & 0.64 \\
Test statistic & 88.86 & 0.61 \\
K-means & 71.94 & 0.32 \\
\hline
\end{tabular}

\subsection{Results on the Vegetated area}

The results of the second region show that changes related to vegetation can be extracted by specific change indices. Fig. 7 indicates that log-ratio on co-polarized response could not extract the destroyed vegetation as obvious as a cross-polarized response which can be inferred that the orientation of vegetated target contributes to change polarizations before returning to the sensor. In Fig. 8, it can be seen that high values in CI are related to volume scattering component of model-based decomposition. The reason behind that is, in vegetated areas volume scattering is a dominant mechanism.

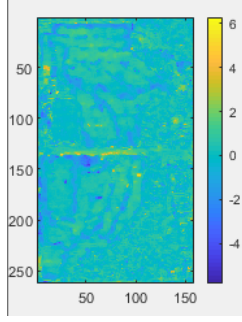

a

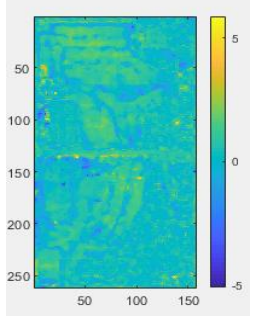

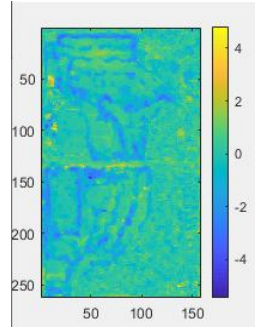

Fig. 7. Change indices in region 2 based on, (a) log-ratio $\mathrm{HH}$, (b) log-ratio VV, (c) $\log$-ratio $\mathrm{VH}$

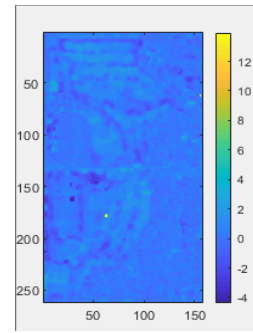

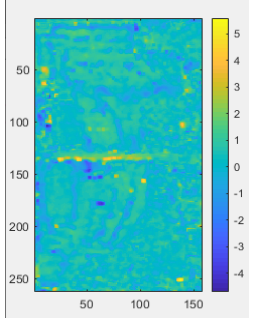

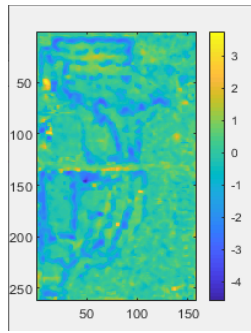

Fig. 8. Change indices in region 2 based on, (a) log-ratio Yamaguchi Odd, (b) log-ratio Yamaguchi Dbl, (c) log-ratio Yamaguchi Vol

Based on the results illustrated in Table 2 and Fig. 9, kernel kmeans clustering has the highest OA. By comparing to the test statistic based method, we can understand that using a test statistic as a change index can improve the discrimination ability. In Fig. 9 (c), we can see that k-means clustering has much false change detection in background resulted in low OA. Results of the test statistic based on Wishart distribution shows that using the covariance matrix of full polarimetry data can extract changes in an acceptable performance.

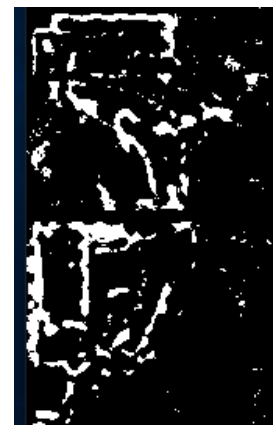

a

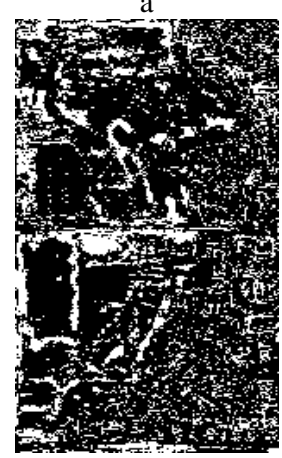

c

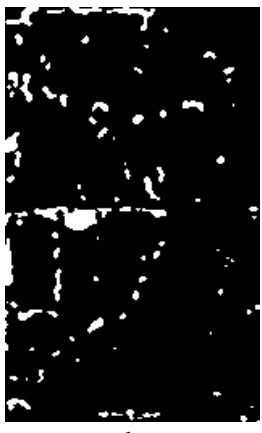

b

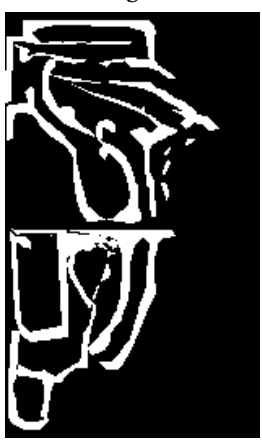

d
Fig. 9. Change detection results in Region 2, (a) proposed method, (b) test statistic based on Wishart distribution, (c) kmeans clustering, (d) ground truth

Table 2 Performance evaluation of the change detection

\begin{tabular}{ccc}
\hline Method & OA (\%) & Kappa \\
\hline Kernel k-means & 86.34 & 0.43 \\
Test statistic & 84.85 & 0.35 \\
K-means & 73.76 & 0.24 \\
\hline
\end{tabular}




\section{CONCLUSION}

This paper presented a method based on change indices and kernel clustering algorithm using an airborne fully polarized Lband sensor in the build-up and vegetated area. In this regard, various change indices on different features were generated, and then kernel k-means clustering was applied to extract changed areas. The evaluation of results demonstrates that the accuracy of the proposed method is relatively better than $\mathrm{k}$-means clustering and a test statistic based methods. In other words, the kernel clustering method shows flexibility to find nonlinear solutions to the problem of change detection. In addition, the results show that generating change indices related to a nature of a region is an important step in extracting changed areas and using a test statistic as a change index improves the performance of the proposed method.

\section{ACKNOWLEDGEMENTS}

The authors acknowledge NASA/JPL-Caltech for UAVSAR data over the area in San Andreas city, the United States used in this research.

\section{REFERENCES}

Akbari, Vahid, Stian N Anfinsen, Anthony P Doulgeris, and Torbjørn Eltoft. 2013. "The Hotelling-Lawley trace statistic for change detection in polarimetric SAR data under the complex Wishart distribution." In 2013 IEEE International Geoscience and Remote Sensing Symposium-IGARSS, 4162-65. IEEE.

Bazi, Yakoub, Lorenzo Bruzzone, and Farid Melgani. 2005. 'An unsupervised approach based on the generalized Gaussian model to automatic change detection in multitemporal SAR images', IEEE Transactions on Geoscience and Remote Sensing, 43: 874-87.

Bovolo, F., and L. Bruzzone. 2015. 'The Time Variable in Data Fusion: A Change Detection Perspective', IEEE Geoscience and Remote Sensing Magazine, 3: 8-26.

Celik, Turgay. 2009. 'Unsupervised change detection in satellite images using principal component analysis and $\$ \mathrm{k} \$$ means clustering', IEEE Geoscience and Remote Sensing Letters, 6: 772-76.

Conradsen, Knut, Allan Aasbjerg Nielsen, Jesper Schou, and Henning Skriver. 2003. 'A test statistic in the complex Wishart distribution and its application to change detection in polarimetric SAR data', IEEE Transactions on Geoscience and Remote Sensing, 41: 4-19.

Gong, Maoguo, Linzhi Su, Meng Jia, and Weisheng Chen. 2013. 'Fuzzy clustering with a modified MRF energy function for change detection in synthetic aperture radar images', IEEE Transactions on Fuzzy Systems, 22: 98-109.

Kittler, Josef, and John Illingworth. 1986. 'Minimum error thresholding', Pattern recognition, 19: 41-47.

Lee, Jong-Sen, and Eric Pottier. 2009. Polarimetric radar imaging: from basics to applications (CRC press).

Li, H., T. Celik, N. Longbotham, and W. J. Emery. 2015. 'Gabor Feature Based Unsupervised Change Detection of Multitemporal SAR Images Based on Two-Level Clustering', IEEE Geoscience and Remote Sensing Letters, 12: 2458-62.

Melgani, Farid, and Yakoub Bazi. 2006. 'Markovian fusion approach to robust unsupervised change detection in remotely sensed imagery', IEEE Geoscience and Remote Sensing Letters, 3: 457-61.

Moser, Gabriele, and Sebastiano B Serpico. 2006. 'Generalized minimum-error thresholding for unsupervised change detection from SAR amplitude imagery', IEEE Transactions on Geoscience and Remote Sensing, 44: 2972-82.

Otsu, Nobuyuki. 1979. 'A threshold selection method from graylevel histograms', IEEE transactions on systems, man, and cybernetics, 9: 62-66.

Radke, Richard J, Srinivas Andra, Omar Al-Kofahi, and Badrinath Roysam. 2005. 'Image change detection algorithms: a systematic survey', IEEE transactions on image processing, 14: 294-307.

Sezgin, Mehmet, and Bülent Sankur. 2004. 'Survey over image thresholding techniques and quantitative performance evaluation', Journal of Electronic imaging, 13: 146-66.

Shawe-Taylor, John, and Nello Cristianini. 2004. Kernel methods for pattern analysis (Cambridge university press).

Volpi, Michele, Devis Tuia, Gustavo Camps-Valls, and Mikhail Kanevski. 2010. "Unsupervised change detection by kernel clustering." In Image and Signal Processing for Remote Sensing XVI, 78300V. International Society for Optics and Photonics. 